\title{
Strong Convergence Properties and Strong Stability for Weighted Sums of AANA Random Variables
}

\section{Zhiyong Chen, Shuhe Hu, Jimin Ling, and Xuejun Wang}

School of Mathematical Science, Anhui University, Hefei 230601, China

Correspondence should be addressed to Xuejun Wang; 07019@ahu.edu.cn

Received 10 August 2013; Accepted 22 October 2013

Academic Editor: Jaume Giné

Copyright (C) 2013 Zhiyong Chen et al. This is an open access article distributed under the Creative Commons Attribution License, which permits unrestricted use, distribution, and reproduction in any medium, provided the original work is properly cited.

The Khintchine-Kolmogorov-type convergence theorem and three-series theorem for AANA random variables are established. By using these convergence theorems, we obtain convergence results for AANA sequences, which extend the corresponding ones for independent sequences and NA sequences. In addition, we study the strong stability for weighted sums of AANA random variables and obtain some new results, which extend some earlier ones for NA random variables.

\section{Introduction}

Firstly, let us recall some definitions.

Definition 1 (cf. Wu [1]). A sequence $\left\{X_{n}, n \geq 1\right\}$ of random variables is said to be stochastically dominated by a random variable $X$ if there exists a constant $C$ such that

$$
P\left(\left|X_{n}\right|>x\right) \leq C P(|X|>x)
$$

for all $x \geq 0$ and $n \geq 1$.

Definition 2 (cf. Chow and Teicher [2]). A sequence $\left\{Y_{n}, n \geq\right.$ $1\}$ of random variables is said to be strongly stable if there exist two constant sequences $\left\{b_{n}, n \geq 1\right\}$ and $\left\{d_{n}, n \geq\right.$ $1\}$ with $0<b_{n} \uparrow \infty$ such that

$$
b_{n}^{-1} Y_{n}-d_{n} \longrightarrow 0 \quad \text { a.s. }
$$

Definition 3 (cf. Wu [1]). A function $l(x)>0(x>0)$ is said to be quasimonotonically increasing function if there exist $x_{0}>0$ and constant $C>0$ with $\forall t \geq x \geq x_{0}$ such that $l(t) \geq C l(x)$. A function $l(x)>0(x>0)$ is said to be quasimonotonically decreasing function if there exist $x_{0}>$ 0 and constant $C>0$ with $\forall t \geq x \geq x_{0}$ such that $l(t) \leq$ $C l(x)$.
Definition 4 (cf. Wu [1]). A real-valued function $l(x)$, positive and measurable on $(0, \infty)$, is said to be slowly varying if

$$
\lim _{x \rightarrow \infty} \frac{l(\lambda x)}{l(x)}=1
$$

for each $\lambda>0$.

Definition 5 (cf. Joag-Dev and Proschan [3]). A finite collection of random variables $X_{1}, X_{2}, \ldots, X_{n}$ is said to be negatively associated (NA, in short) if for every pair of disjoint subsets $A_{1}, A_{2}$ of $\{1,2, \ldots, n\}$,

$$
\operatorname{Cov}\left\{f\left(X_{i}: i \in A_{1}\right), g\left(X_{j}: j \in A_{2}\right)\right\} \leq 0,
$$

whenever $f$ and $g$ are coordinatewise nondecreasing such that this covariance exists. An infinite sequence $\left\{X_{n}, n \geq\right.$ $1\}$ is NA if every finite subcollection is NA.

Definition 6 (cf. Chandra and Ghosal [4]). A sequence $\left\{X_{n}, n \geq 1\right\}$ of random variables is called asymptotically almost negatively associated (AANA) if there exists a nonnegative sequence $q(n) \rightarrow 0$ as $n \rightarrow \infty$ such that

$$
\begin{aligned}
& \operatorname{Cov}\left\{f\left(X_{n}\right), g\left(X_{n+1}, X_{n+2}, \ldots, X_{n+k}\right)\right\} \\
& \quad \leq q(n)\left[\operatorname{Var}\left(f\left(X_{n}\right)\right) \operatorname{Var}\left(g\left(X_{n+1}, X_{n+2}, \ldots, X_{n+k}\right)\right)\right]^{1 / 2}
\end{aligned}
$$


for all $n, k \geq 1$ and for all coordinatewise nondecreasing continuous functions $f$ and $g$ whenever the variances exist.

Obviously, the family of AANA sequences contains NA (in particular, independent) sequences (taking $q(n)=0, n \geq$ 1) and some more sequences of random variables which do not much deviates from being NA. An example of an AANA sequence which is not NA was introduced by Chandra and Ghosal [5].

Since the concept of AANA random variables was introduced by Chandra and Ghosal [4], many applications have been found. For example, Chandra and Ghosal [4] derived the Kolmogorov-type inequality and the strong law of large numbers of Marcinkiewicz-Zygmund, Chandra and Ghosal [5] obtained the almost sure convergence of weighted averages, Ko et al. [6] studied the Hájek-Rènyi type inequality, and Wang et al. [7] established the law of the iterated logarithm for product sums. Yuan and An [8] established Rosenthal-type inequalities for maximum partial sums of AANA sequences. Wang et al. [9] studied some convergence properties for AANA sequence. Wang et al. [10] generalized and improved the results of Ko et al. [6] and studied the large deviation and Marcinkiewicz-type strong law of large numbers for AANA sequences. Yang et al. [11] investigated the complete convergence of moving average process for AANA sequence. $\mathrm{Hu}$ et al. [12] and Shen and $\mathrm{Wu}[13,14]$ studied strong convergence property for weighted sums of AANA sequence. Wang et al. [15, 16] and Shen et al. [17] obtained some results on complete convergence for AANA sequence, and so forth.

In this paper, we mainly study convergence results for AANA random variables, and the strong stability for weighted sums of AANA random variables, which extend the corresponding ones for independent sequences and NA sequences without necessarily adding extra conditions. The techniques used in the paper are the truncated method, the Khintchine-Kolmogorov-type convergence theorem and three-series theorem for AANA random variables.

Throughout this paper, let $I(A)$ be the indicator function of the set $A$, and $X_{n}^{(c)}=-c I\left(X_{n}<-c\right)+X_{n} I\left(\left|X_{n}\right| \leq c\right)+$ $c I\left(X_{n}>c\right)$ for some $c>0 . a_{n}=O\left(b_{n}\right)$ denotes that there exists a positive constant $C$ such that $\left|a_{n} / b_{n}\right| \leq C$. The symbol $C$ represents a positive constant which may be different in various places. The main results of this paper depend on the following lemmas.

Lemma 7 (cf. Yuan and An [8]). Let $\left\{X_{n}, n \geq 1\right\}$ be a sequence of $A A N A$ random variables with mixing coefficients $\{q(n), n \geq 1\}$, and let $f_{1}, f_{2}, \ldots$ be all nondecreasing (or nonincreasing) continuous functions; then $\left\{f_{n}\left(X_{n}\right), n \geq 1\right\}$ is still a sequence of AANA random variables with mixing coefficients $\{q(n), n \geq 1\}$.

Lemma 8 (cf. Wang et al. [9]). Let $1<p \leq 2$ and $\left\{X_{n}, n \geq\right.$ $1\}$ be a sequence of AANA random variables with mixing coefficients $\{q(n), n \geq 1\}$. Assume that $E X_{n}=0$ for all $n \geq 1$ and $\sum_{n=1}^{\infty} q^{2}(n)<\infty$; then there exists a positive constant $C_{p}$ depending only on $p$ such that

$$
E\left(\max _{1 \leq j \leq n}\left|\sum_{i=1}^{j} X_{i}\right|^{p}\right) \leq C_{p} \sum_{i=1}^{n} E\left|X_{i}\right|^{p}
$$

for all $n \geq 1$, where $C_{p}=2^{p}\left[2^{2-p} p+(6 p)^{p}\left(\sum_{n=1}^{\infty} q^{2}(n)\right)^{(p-1)}\right]$.

By Lemmas 7 and 8, we can get the following KhintchineKolmogorov-type convergence theorem and three series theorem for AANA sequences, which can be applied to prove the main results of the paper. The proofs are standard, so we omit them.

Corollary 9 (Khintchine-Kolmogorov-type convergence theorem). Let $\left\{X_{n}, n \geq 1\right\}$ be a sequence of $A A N A$ random variables with mixing coefficients $\{q(n), n \geq 1\}$ and $\sum_{n=1}^{\infty} q^{2}(n)<$ œ. If

$$
\sum_{n=1}^{\infty} \operatorname{Var} X_{n}<\infty
$$

then $\sum_{n=1}^{\infty}\left(X_{n}-E X_{n}\right)$ converges almost surely.

Corollary 10 (three-series theorem for AANA random variables). Let $\left\{X_{n}, n \geq 1\right\}$ be a sequence of AANA random variables with mixing coefficients $\{q(n), n \geq 1\}$ and $\sum_{n=1}^{\infty} q^{2}(n)<$ $\infty$. Assume that for some $c>0$,

$$
\begin{gathered}
\sum_{n=1}^{\infty} P\left(\left|X_{n}\right|>c\right)<\infty, \quad \sum_{n=1}^{\infty} E X_{n}^{(c)} \text { converges } \\
\sum_{n=1}^{\infty} \operatorname{Var} X_{n}^{(c)}<\infty
\end{gathered}
$$

Then, $\sum_{n=1}^{\infty} X_{n}$ converges almost surely.

Remark 11. Since NA implies AANA, Corollaries 9 and 10 extend corresponding results for NA random variables (see Matula [18]) to AANA random variables without adding any extra condition.

Lemma 12 (cf. Wu [19] or Shen [20]). Let $\left\{X_{n}, n \geq 1\right\}$ be a sequence of random variables which is stochastically dominated by a random variable $X$. For any $\alpha>0$ and $b>0$, the following two statements hold:

$$
\begin{gathered}
E\left|X_{n}\right|^{\alpha} I\left(\left|X_{n}\right| \leq b\right) \leq C_{1}\left[E|X|^{\alpha} I(|X| \leq b)+b^{\alpha} P(|X|>b)\right] \\
E\left|X_{n}\right|^{a} I\left(\left|X_{n}\right|>b\right) \leq C_{2} E|X|^{\alpha} I(|X|>b)
\end{gathered}
$$

where $C_{1}$ and $C_{2}$ are positive constants.

Lemma 13 (cf. Wu [1]). Let $h(x)>0$ be a slowly varying function; then for any $\delta>0, x^{\delta} h(x)$ is a quasimonotonically increasing function and $x^{-\delta} h(x)$ is a quasimonotonically decreasing function. 


\section{Strong Convergence Properties of Weighted Sums for AANA Sequence}

Theorem 14. Let $\left\{X_{n}, n \geq 1\right\}$ be a sequence of $A A N A$ random variables with $\sum_{n=1}^{\infty} q^{2}(n)<\infty$. Assume that $\left\{g_{n}(x), n \geq 1\right\}$ is a sequence of even functions defined on $R$. For each $n \geq 1, g_{n}(x)$ is a positive and nondecreasing function in $(0, \infty)$ and satisfies one of the following conditions:

(i) for some $0<r \leq 1, x^{r} / g_{n}(x)$ is a nondecreasing function in $(0, \infty)$;

(ii) for some $1<r \leq 2, x / g_{n}(x)$ and $g_{n}(x) / x^{r}$ are nonincreasing functions in $(0, \infty)$; furthermore, assume that $E X_{n}=0$ for each $n \geq 1$.

For any positive number sequence $\left\{a_{n}, n \geq 1\right\}$ with $a_{n} \uparrow$ $\infty$ such that

$$
\sum_{n=1}^{\infty} \frac{E g_{n}\left(X_{n}\right)}{g_{n}\left(a_{n}\right)}<\infty
$$

then $\sum_{n=1}^{\infty} X_{n} / a_{n}$ converges a.s., and

$$
a_{n}^{-1} \sum_{i=1}^{n} X_{i} \longrightarrow 0 \quad \text { a.s., as } n \longrightarrow \infty \text {. }
$$

Proof. For each $n \geq 1$, denote

$$
\begin{aligned}
X_{n}^{\left(a_{n}\right)}= & -a_{n} I\left(X_{n}<-a_{n}\right)+X_{n} I\left(\left|X_{n}\right| \leq a_{n}\right) \\
& +a_{n} I\left(X_{n}>a_{n}\right) .
\end{aligned}
$$

By Lemma 7, we can see that, for fixed $n \geq 1,\left\{X_{n}^{\left(a_{n}\right)}\right\}$ is still a sequence of AANA random variables. So by Corollary 10 in order to prove (11), we need only to prove the convergence of three series of (8), where $c=1$.

Firstly, we prove that $\sum_{n=1}^{\infty} P\left(\left|X_{n} / a_{n}\right|>1\right)<\infty$ under condition (i) or (ii).

For each $n \geq 1$, if $g_{n}(x)$ satisfies condition (i), noting that $\left\{g_{n}(x), n \geq 1\right\}$ is a sequence of positive and nondecreasing even function in $(0,+\infty)$. Combining Markov's inequality with (10), it follows that

$$
\begin{aligned}
\sum_{n=1}^{\infty} P\left(\left|\frac{X_{n}}{a_{n}}\right|>1\right) & \leq \sum_{n=1}^{\infty} P\left(g_{n}\left(X_{n}\right)>g_{n}\left(a_{n}\right)\right) \\
& \leq \sum_{n=1}^{\infty} \frac{E g_{n}\left(X_{n}\right)}{g_{n}\left(a_{n}\right)}<\infty .
\end{aligned}
$$

If $g_{n}(x)$ satisfies condition (ii), it is easy to prove that (13) also holds.

Secondly, we will show $\sum_{n=1}^{\infty} \operatorname{Var}\left(X_{n}^{\left(a_{n}\right)} / a_{n}\right)<\infty$.

If $g_{n}(x)$ satisfies (i), when $|x| \leq a_{n}$, we have $\left(|x|^{r} / g_{n}(x)\right)$ $\leq\left(a_{n}^{r} / g_{n}\left(a_{n}\right)\right)$, which implies that

$$
\frac{|x|^{r}}{a_{n}^{r}} \leq \frac{g_{n}(x)}{g_{n}\left(a_{n}\right)}, \quad \frac{x^{2}}{a_{n}^{2}} \leq \frac{\left(g_{n}(x)\right)^{2 / r}}{\left(g_{n}\left(a_{n}\right)\right)^{2 / r}} .
$$

Note that $\left\{g_{n}(x), n \geq 1\right\}$ is a sequence of positive and nondecreasing functions in $(0,+\infty)$, so $0 \leq\left(g_{n}(x) / g_{n}\left(a_{n}\right)\right) \leq$ 1 when $|x| \leq a_{n}$. Consequently,

$$
\frac{x^{2}}{a_{n}^{2}} \leq\left(\frac{g_{n}(x)}{g_{n}\left(a_{n}\right)}\right)^{2 / r} \leq \frac{g_{n}(x)}{g_{n}\left(a_{n}\right)}, \quad \text { for } 0<\mathrm{r} \leq 1
$$

On the other hand, if $g_{n}(x)$ satisfies condition (ii), then we can also get that

$$
\frac{x^{2}}{a_{n}^{2}} \leq\left(\frac{g_{n}(x)}{g_{n}\left(a_{n}\right)}\right)^{2 / r} \leq \frac{g_{n}(x)}{g_{n}\left(a_{n}\right)}, \quad \text { for } 1<\mathrm{r} \leq 2
$$

Therefore, whether even function $g_{n}(x)$ satisfies condition (i) or (ii), we can obtain

$$
\begin{aligned}
\operatorname{Var}\left(\frac{X_{n}^{\left(a_{n}\right)}}{a_{n}}\right) \leq & E\left(\frac{X_{n}^{\left(a_{n}\right)}}{a_{n}}\right)^{2} \\
= & E\left(\frac{X_{n}^{2}}{a_{n}^{2}} I\left(\left|X_{n}\right| \leq a_{n}\right)\right)+E I\left(\left|X_{n}\right|>a_{n}\right) \\
\leq & E\left(\frac{g_{n}\left(X_{n}\right)}{g_{n}\left(a_{n}\right)} I\left(\left|X_{n}\right| \leq a_{n}\right)\right) \\
& +E\left(\frac{g_{n}\left(X_{n}\right)}{g_{n}\left(a_{n}\right)} I\left(\left|X_{n}\right|>a_{n}\right)\right)=\frac{E g_{n}\left(X_{n}\right)}{g_{n}\left(a_{n}\right)} .
\end{aligned}
$$

Therefore, it follows from (10) that

$$
\sum_{n=1}^{\infty} \operatorname{Var}\left(\frac{X_{n}^{\left(a_{n}\right)}}{a_{n}}\right) \leq \sum_{n=1}^{\infty} \frac{E g_{n}\left(X_{n}\right)}{g_{n}\left(a_{n}\right)}<\infty
$$

Finally, we prove that $\sum_{n=1}^{\infty} E\left|X_{n}^{\left(a_{n}\right)} / a_{n}\right|<\infty$.

If $g_{n}(x)$ satisfies condition (i), when $|x| \leq a_{n}$, we have $\left(|x| / a_{n}\right) \leq\left(|x|^{r} / a_{n}^{r}\right)$, for $0<r \leq 1$. It follows that

$$
\begin{aligned}
\left|E\left(\frac{X_{n}^{\left(a_{n}\right)}}{a_{n}}\right)\right| \leq & E\left(\frac{\left|X_{n}\right|}{a_{n}} I\left(\left|X_{n}\right| \leq a_{n}\right)\right)+E I\left(\left|X_{n}\right|>a_{n}\right) \\
\leq & E\left(\frac{\left|X_{n}\right|^{r}}{a_{n}^{r}} I\left(\left|X_{n}\right| \leq a_{n}\right)\right) \\
& +E\left(\frac{g_{n}\left(X_{n}\right)}{g_{n}\left(a_{n}\right)} I\left(\left|X_{n}\right|>a_{n}\right)\right) \\
\leq & E\left(\frac{g_{n}\left(X_{n}\right)}{g_{n}\left(a_{n}\right)} I\left(\left|X_{n}\right| \leq a_{n}\right)\right) \\
& +E\left(\frac{g_{n}\left(X_{n}\right)}{g_{n}\left(a_{n}\right)} I\left(\left|X_{n}\right|>a_{n}\right)\right) \\
= & \frac{E g_{n}\left(X_{n}\right)}{g_{n}\left(a_{n}\right)} .
\end{aligned}
$$


If $g_{n}(x)$ satisfies condition (ii), then by the fact that $E X_{n}=0$ and $x / g_{n}(x)$ is a nonincreasing function in $(0, \infty)$, we get

$$
\begin{aligned}
\left|E\left(\frac{X_{n}^{\left(a_{n}\right)}}{a_{n}}\right)\right| \leq & \left|E\left(\frac{X_{n}}{a_{n}} I\left(\left|X_{n}\right| \leq a_{n}\right)\right)\right|+E I\left(\left|X_{n}\right|>a_{n}\right) \\
= & \left|E\left(\frac{X_{n}}{a_{n}} I\left(\left|X_{n}\right|>a_{n}\right)\right)\right|+E I\left(\left|X_{n}\right|>a_{n}\right) \\
\leq & E\left(\frac{\left|X_{n}\right|}{a_{n}} I\left(\left|X_{n}\right|>a_{n}\right)\right)+E I\left(\left|X_{n}\right|>a_{n}\right) \\
\leq & E\left(\frac{g_{n}\left(X_{n}\right)}{g_{n}\left(a_{n}\right)} I\left(\left|X_{n}\right|>a_{n}\right)\right) \\
& +E\left(\frac{g_{n}\left(X_{n}\right)}{g_{n}\left(a_{n}\right)} I\left(\left|X_{n}\right|>a_{n}\right)\right) \\
\leq & 2 \frac{E g_{n}\left(X_{n}\right)}{g_{n}\left(a_{n}\right)} .
\end{aligned}
$$

Therefore, whether $g_{n}(x)$ satisfies condition (i) or (ii), it also follows from (10) that

$$
\sum_{n=1}^{\infty} E\left|\frac{X_{n}^{\left(a_{n}\right)}}{a_{n}}\right|<\infty
$$

The proof of Theorem 14 is completed by (13), (18), and (21).

Corollary 15. Let $\left\{X_{n}, n \geq 1\right\}$ be a sequence of AANA random variables with $\sum_{n=1}^{\infty} q^{2}(n)<\infty$, and let $\left\{a_{n}, n \geq 1\right\}$ be a sequence of positive numbers with $a_{n} \uparrow \infty$. There exists some $0<p \leq 2$ such that

$$
\sum_{n=1}^{\infty} \frac{E\left|X_{n}\right|^{p}}{a_{n}^{p}}<\infty .
$$

If $1<p \leq 2$, we further assume that $E X_{n}=0$. Then, (11) holds.

Proof. We take $g_{n}(x)=|x|^{r}, x \in(-\infty, \infty)$. If $0<r \leq 1$, one can find that $x^{r} / g_{n}(x) \equiv 1$ for $x \geq 0$. So condition (i) of Theorem 14 is satisfied. If $1<r \leq 2$, we have that $x / g_{n}(x)=$ $x^{1-r}$ and $g_{n}(x) / x^{r} \equiv 1$ for $x \geq 0$. Thus, condition (ii) of Theorem 14 is satisfied. Consequently, the desired result (11) follows from Theorem 14 immediately.

Remark 16. If taking $r=1$ in (i) and $r=2$ in (ii), Theorem 14 and Corollary 15 extend the corresponding ones for NA random variables (see Gan [21]) to AANA random variables.

Theorem 17. Let $1<\alpha<2$ and $\left\{X_{n}, n \geq 1\right\}$ be a sequence of AANA random variables with $\sum_{n=1}^{\infty} q^{2}(n)<\infty$ and identical distribution

$$
P\left(\left|X_{1}\right|>x\right)= \begin{cases}L(x) x^{-\alpha}, & x \geq 1 \\ 1, & x<1\end{cases}
$$

where $L(x)$ is a slowly varying function. Let $\left\{a_{n}, n \geq\right.$ $1\}$ and $\left\{b_{n}, n \geq 1\right\}$ be sequences of positive constants satisfying $0<b_{n} \uparrow \infty$. Denote $c_{n}=b_{n} / a_{n}$ for each $n \geq 1$. Assume that

$$
\sum_{n=1}^{\infty} P\left(\left|X_{n}\right|>c_{n}\right)<\infty
$$

then

$$
b_{n}^{-1} \sum_{k=1}^{n} a_{k} X_{k} \longrightarrow 0 \quad \text { a.s., as } n \longrightarrow \infty \text {. }
$$

Proof. Since (23) and (24) imply that $c_{k} \geq 1$ for all sufficiently large $k$. Without loss of generality, we assume $c_{k} \geq 1$ for all $k \geq 1$. that

By Borel-Cantelli Lemma, it is easily seen that (24) implies

$$
\sum_{k=1}^{n} a_{k} X_{k} I\left(\left|X_{k}\right|>c_{k}\right)=o\left(b_{n}\right) \quad \text { a.s. }
$$

Denote

$$
\begin{aligned}
Y_{k}= & -c_{k} I\left(X_{k}<-c_{k}\right)+X_{k} I\left(\left|X_{k}\right| \leq c_{k}\right) \\
& +c_{k} I\left(X_{k}>c_{k}\right), \quad k \geq 1 ;
\end{aligned}
$$

thus, $\left\{Y_{k}, k \geq 1\right\}$ is still AANA from Lemma 7. It is easy to check that

$$
\begin{aligned}
\sum_{k=1}^{n} a_{k} X_{k}= & \sum_{k=1}^{n} a_{k}\left(Y_{k}-E Y_{k}\right)+\sum_{k=1}^{n} a_{k} E Y_{k} \\
& +\sum_{k=1}^{n} a_{k} c_{k}\left(I\left(X_{k}<-c_{k}\right)-I\left(X_{k}>c_{k}\right)\right) \\
& +\sum_{k=1}^{n} a_{k} X_{k} I\left(\left|X_{k}\right|>c_{k}\right) .
\end{aligned}
$$

In order to show that $b_{n}^{-1} \sum_{k=1}^{n} a_{k} X_{k} \rightarrow 0$ a.s., we only need to show that the first three terms above are $o\left(b_{n}\right)$ or $o\left(b_{n}\right)$ a.s.

By $C_{r}$ inequality, Theorem $1 \mathrm{~b}$ in [22, page 281] (or see Adler [23]) and (24), we can get

$$
\begin{aligned}
& \sum_{k=1}^{\infty} \operatorname{Var}\left(\frac{Y_{k}}{c_{k}}\right) \\
& \quad \leq \sum_{k=1}^{\infty} c_{k}^{-2} E Y_{k}^{2} \\
& \quad \leq 3 \sum_{k=1}^{\infty} c_{k}^{-2} E\left[c_{k}^{2} I\left(\left|X_{k}\right|>c_{k}\right)+X_{k}^{2} I\left(\left|X_{k}\right| \leq c_{k}\right)\right] \\
& \quad=3 \sum_{k=1}^{\infty} P\left(\left|X_{k}\right|>c_{k}\right)+3 \sum_{k=1}^{\infty} c_{k}^{-2} E X_{k}^{2} I\left(\left|X_{k}\right| \leq c_{k}\right) \\
& \quad \leq C+6 \sum_{k=1}^{\infty} c_{k}^{-2} \int_{0}^{c_{k}} t P\left(\left|X_{k}\right|>t\right) d t
\end{aligned}
$$




$$
\begin{aligned}
& \leq C+C \sum_{k=1}^{\infty} L\left(c_{k}\right) c_{k}^{-\alpha} \\
& \leq C+C \sum_{k=1}^{\infty} P\left(\left|X_{k}\right|>c_{k}\right)<\infty .
\end{aligned}
$$

It follows from Corollary 9 and Kronecker's lemma that

$$
\sum_{k=1}^{n} a_{k}\left(Y_{k}-E Y_{k}\right)=o\left(b_{n}\right) \text { a.s. }
$$

By (24) again,

$$
\begin{gathered}
\sum_{k=1}^{\infty} E\left|\frac{a_{k} c_{k}\left(I\left(X_{k}<-c_{k}\right)-I\left(X_{k}>c_{k}\right)\right)}{b_{k}}\right| \\
\leq \sum_{k=1}^{\infty} E\left(I\left(X_{k}<-c_{k}\right)+I\left(X_{k}>c_{k}\right)\right) \\
\quad=\sum_{k=1}^{\infty} P\left(\left|X_{k}\right|>c_{k}\right)<\infty
\end{gathered}
$$

which implies that

$$
\sum_{k=1}^{\infty} \frac{a_{k} c_{k}\left(I\left(X_{k}<-c_{k}\right)-I\left(X_{k}>c_{k}\right)\right)}{b_{k}} \text { converges a.s. }
$$

By Kronecker's lemma, it follows that

$$
\sum_{k=1}^{n} a_{k} c_{k}\left(I\left(X_{k}<-c_{k}\right)-I\left(X_{k}>c_{k}\right)\right)=o\left(b_{n}\right) \quad \text { a.s. }
$$

By Theorem $1 b$ in [22, page 281] (or see Adler [23]) and (24) again, we have

$$
\begin{aligned}
& \sum_{k=1}^{\infty}\left|\frac{a_{k} E Y_{k}}{b_{k}}\right| \\
& \quad \leq \sum_{k=1}^{\infty} c_{k}^{-1}\left[c_{k} P\left(\left|X_{k}\right|>c_{k}\right)+E\left|X_{k}\right| I\left(\left|X_{k}\right|>c_{k}\right)\right] \\
& \quad=\sum_{k=1}^{\infty} P\left(\left|X_{k}\right|>c_{k}\right)+\sum_{k=1}^{\infty} c_{k}^{-1} E\left|X_{k}\right| I\left(\left|X_{k}\right|>c_{k}\right) \\
& \quad=2 \sum_{k=1}^{\infty} P\left(\left|X_{k}\right|>c_{k}\right)+\sum_{k=1}^{\infty} c_{k}^{-1} \int_{c_{k}}^{\infty} P\left(\left|X_{k}\right|>t\right) d t \\
& \leq C+C \sum_{k=1}^{\infty} c_{k}^{-1} \int_{c_{k}}^{\infty} L(t) t^{-\alpha} d t \\
& \leq C+C \sum_{k=1}^{\infty} L\left(c_{k}\right) c_{k}^{-\alpha} \\
& \leq C+C \sum_{k=1}^{\infty} P\left(\left|X_{k}\right|>c_{k}\right)<\infty,
\end{aligned}
$$

which implies that

$$
\sum_{k=1}^{\infty} \frac{a_{k} E Y_{k}}{b_{k}} \text { converges. }
$$

By Kronecker's Lemma, it follows that

$$
\sum_{k=1}^{n} a_{k} E Y_{k}=o\left(b_{n}\right)
$$

Hence, the desired result (25) follows from (26)-(36) immediately.

Remark 18. Theorem 17 generalizes and extends the corresponding one for NA random variables (see Wang et al. [24]) to AANA random variables.

Theorem 19. Let $1<r<2$ and $\left\{X_{n}, n \geq 1\right\}$ a sequence of mean zero AANA random variables with $\sum_{n=1}^{\infty} q^{2}(n)<\infty$, which is stochastically dominated by a random variable $X$. Let $\left\{a_{n}, n \geq 1\right\}$ be a sequence of positive constants satisfying $A_{n} \doteq$ $\sum_{k=1}^{n} a_{k} \uparrow \infty$. Denote $c_{n}=A_{n} / a_{n}$ for each $n \geq 1$. Assume that

$$
E|X|^{r}<\infty
$$

$$
N(n) \doteq \operatorname{Card}\left\{\mathrm{i}: \mathrm{c}_{\mathrm{i}} \leq \mathrm{n}\right\}=\mathrm{O}\left(\mathrm{n}^{\mathrm{r}}\right), \quad \mathrm{n} \geq 1
$$

then

$$
A_{n}^{-1} \sum_{k=1}^{n} a_{k} X_{k} \longrightarrow 0 \text { a.s., } \quad \text { as } n \longrightarrow \infty \text {. }
$$

Proof. Let $N(0)=0$ and denote

$$
\begin{aligned}
X_{n}^{\left(c_{n}\right)}= & -c_{n} I\left(X_{n}<-c_{n}\right)+X_{n} I\left(\left|X_{n}\right| \leq c_{n}\right) \\
& +c_{n} I\left(X_{n}>c_{n}\right), \quad n \geq 1 .
\end{aligned}
$$

It follows by (37) that

$$
\begin{aligned}
& \sum_{i=1}^{\infty} P\left(X_{i} \neq X_{i}^{\left(c_{i}\right)}\right) \\
& \quad=\sum_{i=1}^{\infty} P\left(\left|X_{i}\right|>c_{i}\right)=\sum_{j=1}^{\infty} \sum_{c_{i} \leq j<c_{i}+1} P\left(\left|X_{i}\right|>c_{i}\right) \\
& \quad \leq C \sum_{j=1}^{\infty} \sum_{j-1<c_{i} \leq j} P(|X|>j-1) \\
& \quad=C \sum_{j=1}^{\infty}(N(j)-N(j-1)) P(|X|>j-1) \\
& =C \sum_{j=1}^{\infty}(N(j)-N(j-1)) \sum_{n=j}^{\infty} P(n-1<|X| \leq n) \\
& =C \sum_{n=1}^{\infty} \sum_{j=1}^{n}(N(j)-N(j-1)) P(n-1<|X| \leq n) \\
& \quad \leq C \sum_{n=1}^{\infty} n^{r} P(n-1<|X| \leq n) \leq C E|X|^{r}<\infty .
\end{aligned}
$$


By the equality above and Borel-Cantelli lemma, we can get $P\left(X_{i} \neq X_{i}^{\left(c_{i}\right)}\right.$, i.o. $)=0$. Therefore, in order to prove (38), we only need to prove that

$$
A_{n}^{-1} \sum_{i=1}^{n} a_{i} X_{i}^{\left(c_{i}\right)} \longrightarrow 0 \quad \text { a.s., } \mathrm{n} \longrightarrow \infty \text {. }
$$

By $C_{r}$ inequality, Lemma 12 , and (37) again,

$$
\begin{aligned}
\sum_{k=1}^{\infty} \operatorname{Var}\left(\frac{a_{k} X_{k}^{\left(c_{k}\right)}}{A_{k}}\right) & \\
\leq & \sum_{k=1}^{\infty} c_{k}{ }^{-2} E\left(X_{k}^{\left(c_{k}\right)}\right)^{2} \\
\leq & 3 \sum_{k=1}^{\infty} c_{k}{ }^{-2} E\left[c_{k}^{2} I\left(\left|X_{k}\right|>c_{k}\right)+X_{k}^{2} I\left(\left|X_{k}\right| \leq c_{k}\right)\right] \\
\leq & C \sum_{k=1}^{\infty} P\left(|X|>c_{k}\right)+C \sum_{k=1}^{\infty} c_{k}^{-2} E X^{2} I\left(|X| \leq c_{k}\right) \\
\leq & C+C \sum_{j=1}^{\infty} \sum_{j-1<c_{k} \leq j} c_{k}^{-2} E X^{2} I\left(|X| \leq c_{k}\right) \\
\leq & C+C \sum_{j=1}^{\infty} \sum_{j-1<c_{k} \leq j} c_{k}^{-2} E X^{2} I(|X| \leq j) \\
\leq & C+C \sum_{j=2}^{\infty}(N(j)-N(j-1))(j-1)^{-2} \\
\leq & C+C E \sum_{k=2}^{\infty} E|X|^{r} I(k-1<|X| \leq k) \\
\leq & C+C \sum_{k=2}^{j} \sum_{j=k}^{\infty} j^{r-3} E X^{2} I(k-1<|X| \leq k) \\
& \times C \sum_{k=1}^{2} I\left(k-1<\mid X \sum_{k=2}^{\infty} \sum_{j=k}^{\infty} N(j)\left((j-1)^{-2}-j^{-2}\right)\right. \\
\leq & C+C \sum_{k=2}^{\infty} \sum_{j=k}^{\infty}(N(j)-N(j-1)) \\
&
\end{aligned}
$$

Hence, by the inequality above, Corollary 9 and Kronecker's lemma, we have

$$
A_{n}^{-1} \sum_{i=1}^{n} a_{i}\left(X_{i}^{\left(c_{i}\right)}-E X_{i}^{\left(c_{i}\right)}\right) \longrightarrow 0 \quad \text { a.s., } \quad \mathrm{n} \longrightarrow \infty .
$$

In order to prove (41), it suffices to prove that

$$
A_{n}^{-1} \sum_{i=1}^{n} a_{i} E X_{i}^{\left(c_{i}\right)} \longrightarrow 0 \quad n \longrightarrow \infty .
$$

Notice that $E X_{n}=0$ for each $n \geq 1$, we have

$$
\left|E X_{n} I\left(\left|X_{n}\right| \leq c_{n}\right)\right|=\left|E X_{n} I\left(\left|X_{n}\right|>c_{n}\right)\right| .
$$

It follows from Lemma 12 and (37) that,

$$
\begin{aligned}
\sum_{k=1}^{\infty}\left|\frac{a_{k} E X_{k}^{\left(c_{k}\right)}}{A_{k}}\right| & \\
\leq & \sum_{k=1}^{\infty}\left[P\left(\left|X_{k}\right|>c_{k}\right)+c_{k}^{-1}\left|E X_{k} I\left(\left|X_{k}\right| \leq c_{k}\right)\right|\right] \\
& =\sum_{k=1}^{\infty}\left[P\left(\left|X_{k}\right|>c_{k}\right)+c_{k}^{-1}\left|E X_{k} I\left(\left|X_{k}\right|>c_{k}\right)\right|\right] \\
& \leq \sum_{k=1}^{\infty} P\left(\left|X_{k}\right|>c_{k}\right)+\sum_{k=1}^{\infty} c_{k}^{-1} E\left|X_{k}\right| I\left(\left|X_{k}\right|>c_{k}\right) \\
& \leq C \sum_{j=1}^{\infty} P\left(|X|>c_{k}\right)+C \sum_{k=1}^{\infty} c_{k}^{-1} E|X| I\left(|X|>c_{k}\right) \\
& \leq C+C \sum_{j=1}^{\infty} \sum_{c_{k} \leq j<c_{k}+1} c_{k}^{-1} E|X| I\left(|X|>c_{k}\right) \\
& \leq C+C \sum_{j=1}^{\infty} \sum_{j-1<c_{k} \leq j} c_{k}^{-1} E|X| I(|X|>j-1) \\
\leq & \leq C+C \sum_{j=2}^{\infty}(N(j)-N(j-1))(j-1)^{-1} \\
& \leq C+C \sum_{k=1}^{\infty} \sum_{j=2}^{\infty} N(j)\left((j-1)^{-1}-j^{-1}\right) \\
& \times \sum_{k=j-1}^{\infty} E|X| I(k<|X| \leq k+1) \\
& \leq C \sum_{k=1}^{\infty} k_{j=2}^{r-1} E|X| I(k<|X| \leq k+1) \\
& \leq j^{r-2} E|X| I(k<|X| \leq k+1) \\
&
\end{aligned}
$$




$$
\begin{aligned}
& \leq C+C \sum_{k=1}^{\infty} E|X|^{r} I(k<|X| \leq k+1) \\
& \leq C+C E|X|^{r}<\infty .
\end{aligned}
$$

By Kronecker's lemma, we can get (44) immediately. The proof is complete.

\section{Strong Stability for Weighted Sums of AANA Sequence}

Theorem 20. Let $\left\{X_{n}, n \geq 1\right\}$ be a sequence of AANA random variables with $\sum_{n=1}^{\infty} q^{2}(n)<\infty$, which is stochastically dominated by a random variable $X$. Let $\left\{a_{n}, n \geq\right.$ $1\}$ and $\left\{b_{n}, n \geq 1\right\}$ be two sequences of positive numbers with $c_{n}=b_{n} / a_{n}$ and $b_{n} \uparrow \infty$. Denote $N(x)=\operatorname{Card}\left\{n: c_{n} \leq\right.$ $x\}, x>0$. If the following conditions are satisfied:

(i) $E N(|X|)<\infty$;

(ii) $\int_{0}^{\infty} t^{p-1} P(|X|>t)\left(\int_{t}^{\infty} y^{-(p+1)} N(y) d y\right) d t<\infty$, for some $p \in[1,2]$,

then there exist $d_{n} \in \mathbf{R}, n=1,2, \ldots$, such that

$$
b_{n}^{-1} \sum_{i=1}^{n} a_{i} X_{i}-d_{n} \longrightarrow 0 \quad \text { a.s. }
$$

Proof. For each $i \geq 1$, denote

$$
X_{i}^{\left(c_{i}\right)}=-c_{i} I\left(X_{i}<-c_{i}\right)+X_{i} I\left(\left|X_{i}\right| \leq c_{i}\right)+c_{i} I\left(X_{i}>c_{i}\right) .
$$

By Definition 1 and conditions (i), we can obtain

$$
\begin{aligned}
& \sum_{i=1}^{\infty} P\left(X_{i} \neq X_{i}^{\left(c_{i}\right)}\right) \\
& \quad=\sum_{i=1}^{\infty} P\left(\left|X_{i}\right|>c_{i}\right) \leq C \sum_{i=1}^{\infty} P\left(|X|>c_{i}\right) \\
& \quad \leq C \sum_{i=1}^{\infty} \int_{0}^{\infty} I\left(c_{i} \leq t\right) d P(|X| \leq t) \\
& \quad=C \int_{0}^{\infty} N(t) d P(|X| \leq t) \\
& \quad=C E N(|X|)<\infty .
\end{aligned}
$$

By Borel-Cantelli lemma for any sequence $\left\{d_{n}, n \geq 1\right\} \subset$ $\mathbf{R}$, with probability 1 , the sequences $\left\{b_{n}^{-1} \sum_{i=1}^{n} a_{i} X_{i}-d_{n}\right\}$ and $\left\{b_{n}^{-1} \sum_{i=1}^{n} a_{i} X_{i}^{\left(c_{i}\right)}-d_{n}\right\}$ converge on the same set and to the same limit. We will prove that $b_{n}^{-1} \sum_{i=1}^{n} a_{i}\left(X_{i}^{\left(c_{i}\right)}-E X_{i}^{\left(c_{i}\right)}\right) \rightarrow 0$ a.s., which implies (6) with $d_{n}=b_{n}^{-1} \sum_{i=1}^{n} a_{i} E X_{i}^{\left(c_{i}\right)}$. According to Lemma 7, $\left\{a_{i}\left(X_{i}^{\left(c_{i}\right)}-E X_{i}^{\left(c_{i}\right)}\right), i \geq 1\right\}$ is a sequence of
AANA random variables with mean zero. By $C_{r}$ inequality and Lemma 12, we have

$$
\begin{aligned}
\sum_{n=1}^{\infty} \frac{E\left|a_{n}\left(X_{n}^{\left(c_{n}\right)}-E X_{n}^{\left(c_{n}\right)}\right)\right|^{p}}{b_{n}^{p}} & \\
\leq & C \sum_{n=1}^{\infty} c_{n}^{-p} E\left(\left|X_{n}\right|^{p} I\left(\left|X_{n}\right| \leq c_{n}\right)\right) \\
& +C \sum_{n=1}^{\infty} c_{n}^{-p} c_{n}^{p} P\left(\left|X_{n}\right|>c_{n}\right) \\
\leq & C \sum_{n=1}^{\infty} c_{n}^{-p}\left[c_{n}^{p} P\left(|X|>c_{n}\right)+E\left(|X|^{p} I\left(|X| \leq c_{n}\right)\right)\right] \\
& +C \sum_{n=1}^{\infty} P\left(|X|>c_{n}\right) \\
\leq & C E N(|X|)+C \sum_{n=1}^{\infty} c_{n}^{-p} \int_{0}^{c_{n}} t^{p-1} P(|X|>t) d t .
\end{aligned}
$$

Notice that

$$
\begin{aligned}
& \sum_{n=1}^{\infty} c_{n}^{-p} \int_{0}^{c_{n}} t^{p-1} P(|X|>t) d t \\
& \quad=\int_{0}^{\infty} t^{p-1} P(|X|>t) \sum_{n: c_{n} \geq t} c_{n}^{-p} d t \\
& \quad \leq C \int_{0}^{\infty} t^{p-1} P(|X|>t)\left(\int_{t}^{\infty} y^{-(p+1)} N(y) d y\right) d t,
\end{aligned}
$$

where the last inequality follows from the fact that for $t>0$

$$
\begin{aligned}
& \sum_{n: c_{n} \geq t} c_{n}^{-p} \\
& \quad=\lim _{u \rightarrow \infty} \sum_{n: t \leq c_{n} \leq u} c_{n}^{-p}=\lim _{u \rightarrow \infty} \int_{t}^{u} y^{-p} d N(y) \\
& \quad=\lim _{u \rightarrow \infty}\left(u^{-p} N(u)-t^{-p} N(t)+p \int_{t}^{u} y^{-(p+1)} N(y) d y\right), \\
& \varlimsup_{u \rightarrow \infty}\left(u^{-p} N(u)\right) \\
& \quad \leq \varlimsup_{u \rightarrow \infty}\left(p \int_{u}^{\infty} y^{-(p+1)} N(y) d y\right) \\
& \quad \leq p \int_{t}^{\infty} y^{-(p+1)} N(y) d y .
\end{aligned}
$$

By (50), (51) and condition (i), (ii), we can get that

$$
\sum_{n=1}^{\infty} \frac{E\left|a_{n}\left(X_{n}^{\left(c_{n}\right)}-E X_{n}^{\left(c_{n}\right)}\right)\right|^{p}}{b_{n}^{p}}<\infty .
$$


Therefore, it follows from (53) and Corollary 15 that

$$
b_{n}^{-1} \sum_{i=1}^{n} a_{i}\left(X_{i}^{\left(c_{i}\right)}-E X_{i}^{\left(c_{i}\right)}\right) \longrightarrow 0, \text { a.s. }
$$

The proof is complete.

Corollary 21. Suppose that the conditions of Theorem 20 are satisfied and $E X_{n}=0$ for each $n \geq 1$. If $\int_{1}^{\infty} E N(|X| / s) d s<$ $\infty$, then $b_{n}^{-1} \sum_{i=1}^{n} a_{i} X_{i} \rightarrow 0$ a.s.

Proof. According to the proof of Theorem 20, we need only to prove that

$$
b_{n}^{-1} \sum_{i=1}^{n} a_{i} E X_{i}^{\left(c_{i}\right)} \longrightarrow 0, \quad \text { as } \mathrm{n} \longrightarrow \infty .
$$

Notice that $E X_{n}=0$ for each $n \geq 1$; then

$$
\begin{aligned}
\left|E X_{i}^{\left(c_{i}\right)}\right| & \mid-E\left(X_{i} I\left(\left|X_{i}\right|>c_{i}\right)\right)-E\left(c_{i} I\left(X_{i}<-c_{i}\right)\right) \\
& \quad+E\left(c_{i} I\left(X_{i}>c_{i}\right)\right) \mid \\
= & \left|E\left(\left(X_{i}-c_{i}\right) I\left(X_{i}>c_{i}\right)\right)+E\left(\left(X_{i}+c_{i}\right) I\left(X_{i}<-c_{i}\right)\right)\right| \\
\leq & E\left(\left(\left|X_{i}\right|+c_{i}\right) I\left(\left|X_{i}\right|>c_{i}\right)\right) \\
\sum_{i=1}^{\infty} & \begin{array}{l}
a_{i}\left|E X_{i}^{\left(c_{i}\right)}\right| \\
b_{i}
\end{array} \\
\leq & \sum_{i=1}^{\infty} c_{i}^{-1} E\left(\left(\left|X_{i}\right|+c_{i}\right) I\left(\left|X_{i}\right|>c_{i}\right)\right) \\
= & \sum_{i=1}^{\infty} P\left(\left|X_{i}\right|>c_{i}\right)+\sum_{i=1}^{\infty} c_{i}^{-1} E\left(\left|X_{i}\right| I\left(\left|X_{i}\right|>c_{i}\right)\right) \\
\leq & C E N(|X|)+\sum_{i=1}^{\infty} c_{i}^{-1} \int_{c_{i}}^{\infty} P\left(\left|X_{i}\right|>t\right) d t \\
\leq & C E N(|X|)+C \int_{1}^{\infty} E N\left(\frac{|X|}{s}\right) d s<\infty \\
= & C E N(|X|)+\int_{1}^{\infty} \sum_{i=1}^{\infty} P\left(\left|X_{i}\right|>s c_{i}\right) d s
\end{aligned}
$$

By Kronecker's lemma, we can get (55) immediately.

Theorem 22. Let $\left\{X_{n}, n \geq 1\right\}$ be a sequence of $A A N A$ random variables with mean zero and $\sum_{n=1}^{\infty} q^{2}(n)<\infty$, which is stochastically dominated by a random variable $X$. Let $\left\{a_{n}, n \geq\right.$ $1\}$ and $\left\{b_{n}, n \geq 1\right\}$ be two sequences of positive numbers with $c_{n}=b_{n} / a_{n}$ and $b_{n} \uparrow \infty$. Denote $N(x)=\operatorname{Card}\left\{n: c_{n} \leq\right.$ $x\}, x>0$. If the following conditions are satisfied:

(i) $\operatorname{EN}(|X|)<\infty$;

(ii) $\int_{1}^{\infty} E N(|X| / s) d s<\infty$;

(iii) $\max _{1 \leq j \leq n} c_{j}^{p} \sum_{i=n}^{\infty} c_{i}^{-p}=O(n)$, for some $p \in[1,2]$, then

$$
b_{n}^{-1} \sum_{i=1}^{n} a_{i} X_{i} \longrightarrow 0 \quad \text { a.s. }
$$

Proof. By (49), condition (i), and Borel-Cantelli lemma, it suffices to prove $b_{n}^{-1} \sum_{i=1}^{\infty} a_{i} X_{i}^{\left(c_{i}\right)} \rightarrow 0$, a.s. So we need only to prove

$$
\begin{aligned}
& b_{n}^{-1} \sum_{i=1}^{n} a_{i}\left(X_{i}^{\left(c_{i}\right)}-E X_{i}^{\left(c_{i}\right)}\right) \longrightarrow 0 \quad \text { a.s., } \\
& b_{n}^{-1} \sum_{i=1}^{n} a_{i} E X_{i}^{\left(c_{i}\right)} \longrightarrow 0, \quad \text { as } \mathrm{n} \longrightarrow \infty .
\end{aligned}
$$

We can get (60) from the proof of Corollary 21. In the following, we prove (59). Put $\varepsilon_{0}=0$ and $\varepsilon_{n}=\max _{1 \leq j \leq n} c_{j}$ for $n \geq$ 1. According to Lemma $7,\left\{a_{i}\left(X_{i}^{\left(c_{i}\right)}-E X_{i}^{\left(c_{i}\right)}\right), i \geq 1\right\}$ is a sequence of AANA random variables with mean zero. By $C_{r}$ inequality and Lemma 12 ,

$$
\begin{aligned}
& \sum_{n=1}^{\infty} \frac{E\left|a_{n}\left(X_{n}^{\left(c_{n}\right)}-E X_{n}^{\left(c_{n}\right)}\right)\right|^{p}}{b_{n}^{p}} \\
& \leq C \sum_{n=1}^{\infty} c_{n}^{-p} E\left(\left|X_{n}\right|^{p} I\left(\left|X_{n}\right| \leq c_{n}\right)\right) \\
& \quad+C \sum_{n=1}^{\infty} P\left(\left|X_{n}\right|>c_{n}\right) \\
& \leq C \sum_{n=1}^{\infty} c_{n}^{-p}\left[c_{n}^{p} P\left(|X|>c_{n}\right)+E\left(|X|^{p} I\left(|X| \leq c_{n}\right)\right)\right] \\
& \quad+C \sum_{n=1}^{\infty} P\left(|X|>c_{n}\right) \\
& \leq C E N(|X|)+C \sum_{n=1}^{\infty} c_{n}^{-p} E\left(|X|^{p} I\left(|X| \leq c_{n}\right)\right) .
\end{aligned}
$$

It is easy to see that

$$
\begin{aligned}
& \sum_{n=1}^{\infty} c_{n}^{-p} E\left(|X|^{p} I\left(|X| \leq c_{n}\right)\right) \\
& \quad \leq \sum_{n=1}^{\infty} c_{n}^{-p} E\left(|X|^{p} I\left(|X| \leq \varepsilon_{n}\right)\right) \\
& \quad=\sum_{n=1}^{\infty} c_{n}^{-p} \sum_{j=1}^{n} E\left(|X|^{p} I\left(\varepsilon_{j-1}<|X| \leq \varepsilon_{j}\right)\right) \\
& \quad \leq \sum_{j=1}^{\infty} P\left(\varepsilon_{j-1}<|X| \leq \varepsilon_{j}\right) \varepsilon_{j}^{p} \sum_{n=j}^{\infty} c_{n}^{-p} \\
& \quad \leq \sum_{j=1}^{\infty} j P\left(\varepsilon_{j-1}<|X| \leq \varepsilon_{j}\right) \\
& \quad \leq C \sum_{n=1}^{\infty} P\left(|X|>\varepsilon_{n-1}\right) \leq C\left(1+\sum_{n=1}^{\infty} P\left(|X|>c_{n}\right)\right) \\
& \quad \leq C(1+E N(|X|))<\infty .
\end{aligned}
$$


Therefore,

$$
\sum_{n=1}^{\infty} \frac{E\left|a_{n}\left(X_{n}^{\left(c_{n}\right)}-E X_{n}^{\left(c_{n}\right)}\right)\right|^{p}}{b_{n}^{p}}<\infty
$$

follows from condition (i), (61) and (62). By Corollary 15 and (63), we can obtain (59) immediately. The proof is complete.

Theorem 23. Let $\left\{X_{n}, n \geq 1\right\}$ be a sequence of $A A N A$ random variables with $\sum_{n=1}^{\infty} q^{2}(n)<\infty$, which is stochastically dominated by a random variable $X$. Let $\left\{a_{n}, n \geq\right.$ $1\}$ and $\left\{b_{n}, n \geq 1\right\}$ be two sequences of positive numbers with $c_{n}=b_{n} / a_{n}$ and $b_{n} \uparrow \infty$. Define $N(x)=\operatorname{Card}\left\{n: c_{n} \leq\right.$ $x\}, R(x)=\int_{x}^{\infty} N(y) y^{-3} d y, x>0$. If

(i) $N(x)<\infty$ for any $x>0$;

(ii) $R(1)=\int_{1}^{\infty} N(y) y^{-3} d y<\infty$;

(iii) $E\left(X^{2} R(|X|)\right)<\infty$,

then there exist $d_{n} \in \mathbf{R}, n=1,2, \ldots$, such that

$$
b_{n}^{-1} \sum_{i=1}^{n} a_{i} X_{i}-d_{n} \longrightarrow 0 \quad \text { a.s. }
$$

Proof. According to Lemma 7, $\left\{X_{i}^{\left(c_{i}\right)}, i \geq 1\right\}$ and $\left\{X_{i}^{\left(c_{i}\right)} / c_{i}, i \geq\right.$ $1\}$ are sequences of AANA random variables.

Since $N(x)$ is nondecreasing, then for any $x>0$

$$
R(x) \geq N(x) \int_{x}^{\infty} y^{-3} d y=\frac{1}{2} x^{-2} N(x),
$$

which implies that $E N(|X|) \leq 2 E\left(X^{2} R(|X|)\right)<\infty$. Therefore,

$$
\begin{aligned}
\sum_{i=1}^{\infty} P\left(X_{i} \neq X_{i}^{\left(c_{i}\right)}\right) & =\sum_{i=1}^{\infty} P\left(\left|X_{i}\right|>c_{i}\right) \\
& \leq C \sum_{i=1}^{\infty} P\left(|X|>c_{i}\right) \leq C E N(|X|)<\infty .
\end{aligned}
$$

By Borel-Cantelli lemma for any sequence $\left\{d_{n}, n \geq 1\right\} \subset$ $\mathbf{R}$, with probability 1 , the sequences $\left\{b_{n}^{-1} \sum_{i=1}^{n} a_{i} X_{i}-d_{n}\right\}$ and $\left\{b_{n}^{-1} \sum_{i=1}^{n} a_{i} X_{i}^{\left(c_{i}\right)}-d_{n}\right\}$ converge on the same set and to the same limit. We will prove that $b_{n}^{-1} \sum_{i=1}^{n} a_{i}\left(X_{i}^{\left(c_{i}\right)}-E X_{i}^{\left(c_{i}\right)}\right) \rightarrow$
0 a.s., which implies the theorem with $d_{n}=b_{n}^{-1} \sum_{i=1}^{n} a_{i} E X_{i}^{\left(c_{i}\right)}$. By $C_{r}$ inequality and Lemma 12 ,

$$
\begin{aligned}
\sum_{n=1}^{\infty} \operatorname{Var}\left(\frac{a_{n} X_{n}^{\left(c_{n}\right)}}{b_{n}}\right) & \\
\leq & \sum_{n=1}^{\infty} c_{n}^{-2} E\left(X_{n}^{\left(c_{n}\right)}\right)^{2} \\
\leq & 3 \sum_{n=1}^{\infty} P\left(\left|X_{n}\right|>c_{n}\right)+3 \sum_{n=1}^{\infty} c_{n}^{-2} E\left(X_{n}^{2} I\left(\left|X_{n}\right| \leq c_{n}\right)\right) \\
\leq & C \sum_{n=1}^{\infty} P\left(|X|>c_{n}\right) \\
& +C \sum_{n=1}^{\infty} c_{n}^{-2}\left[c_{n}^{2} P\left(|X|>c_{n}\right)+E\left(X^{2} I\left(|X| \leq c_{n}\right)\right)\right] \\
\leq & C E N(|X|) \\
& +C \sum_{n=1}^{\infty} c_{n}^{-2} E\left(X^{2} I\left(|X| \leq c_{n}\right)\right) \\
\doteq & C E N(|X|)+C I,
\end{aligned}
$$

$$
\begin{aligned}
I= & \sum_{n: c_{n} \leq 1} c_{n}^{-2} E\left(X^{2} I\left(|X| \leq c_{n}\right)\right) \\
& +\sum_{n: c_{n}>1} c_{n}^{-2} E\left(X^{2} I\left(|X| \leq c_{n}\right)\right) \\
\doteq & I_{1}+I_{2} .
\end{aligned}
$$

Since $N(1)=\operatorname{Card}\left\{n: c_{n} \leq 1\right\} \leq 2 R(1)<\infty$, following from (65) and condition (ii), then we have $I_{1}<\infty$.

$$
\begin{aligned}
I_{2} & =\sum_{n: c_{n}>1} c_{n}^{-2} E\left(X^{2} I\left(|X| \leq c_{n}\right)\right) \\
& =\sum_{k=2}^{\infty} \sum_{(k-1)<c_{n} \leq k} c_{n}^{-2} E\left(X^{2} I\left(|X| \leq c_{n}\right)\right) \\
& \leq \sum_{k=2}^{\infty}(N(k)-N(k-1))(k-1)^{-2} E\left(X^{2} I(|X| \leq k)\right) \\
& \leq \sum_{k=2}^{\infty}(N(k)-N(k-1))(k-1)^{-2} E\left(X^{2} I(|X| \leq 1)\right) \\
& +\sum_{k=2}^{\infty}(N(k)-N(k-1)) \\
& \quad \times(k-1)^{-2} E\left(X^{2} I(1<|X| \leq k)\right) \\
& I_{21}+I_{22} .
\end{aligned}
$$


To prove $I_{2}<\infty$, we need to prove that $I_{21}<\infty$ and $I_{22}<$ $\infty$ :

$$
\begin{aligned}
I_{21} & \leq C \sum_{k=2}^{\infty}(N(k)-N(k-1)) \sum_{j=k-1}^{\infty} j^{-3} \\
& =C \sum_{j=1}^{\infty} j^{-3} \sum_{k=2}^{j+1}(N(k)-N(k-1)) \\
& \leq C \sum_{j=1}^{\infty}(j+1)^{-3} N(j+1) \\
& \leq C \int_{1}^{\infty} y^{-3} N(y) d y<\infty .
\end{aligned}
$$

Since $N(x)$ is nondecreasing and $R(x)$ is nonincreasing, then

$$
\begin{aligned}
I_{22}= & \sum_{k=2}^{\infty}(N(k)-N(k-1))(k-1)^{-2} \\
& \times \sum_{m=2}^{k} E\left(X^{2} I(m-1<|X| \leq m)\right) \\
= & \sum_{m=2}^{\infty} E\left(X^{2} I(m-1<|X| \leq m)\right) \\
& \times \sum_{k=m}^{\infty}(N(k)-N(k-1))(k-1)^{-2} \\
\leq & \sum_{m=2}^{\infty} E\left(X^{2} I(m-1<|X| \leq m)\right) \\
& \times \sum_{k=m}^{\infty} N(k)\left((k-1)^{-2}-k^{-2}\right) \\
\leq & C \sum_{m=2}^{\infty} E\left(X^{2} R(|X|) I(m-1<|X| \leq m)\right) \\
\leq & C \sum_{m=2}^{\infty} E\left(X^{2} I(m-1<|X| \leq m)\right) \\
& \times \sum_{k=m}^{\infty} \int_{k}^{\infty} \int_{k}^{k+1} N(x) x^{-3} d x \\
& C\left(X^{2} I(m-1<|X| \leq m)\right) \\
& \\
& \\
& \\
&
\end{aligned}
$$

By (67)-(71), we can get that

$$
\sum_{n=1}^{\infty} \operatorname{Var}\left(\frac{a_{n} X_{n}^{\left(c_{n}\right)}}{b_{n}}\right)<\infty
$$

Therefore, it follows from Corollary 9 and Kronecker's lemma that

$$
b_{n}^{-1} \sum_{i=1}^{n} a_{i}\left(X_{i}^{\left(c_{i}\right)}-E X_{i}^{\left(c_{i}\right)}\right) \longrightarrow 0 \quad \text { a.s. }
$$

Taking $d_{n}=b_{n}^{-1} \sum_{i=1}^{n} a_{i} E X_{i}^{\left(c_{i}\right)}, n \geq 1$, we can get (64). The proof is complete.

Remark 24. Since NA implies AANA, Theorem 20 extends corresponding result for NA random variable (see Wang et al. [24]) to AANA random variables without adding any extra condition.

Similar to the proof of Corollary 21, we can get the following corollary.

Corollary 25. Let the conditions of Theorem 23 be satisfied and $E X_{n}=0$ for each $n \geq 1$. If $\int_{1}^{\infty} E N(|X| / s) d s<\infty$, then $b_{n}^{-1} \sum_{i=1}^{n} a_{i} X_{i} \rightarrow 0$ a.s.

Corollary 26. Let $\left\{X_{n}, n \geq 1\right\}$ be a sequence of AANA random variables with $\sum_{n=1}^{\infty} q^{2}(n)<\infty$, which is stochastically dominated by a random variable $X$. Let $\left\{a_{n}, n \geq\right.$ $1\}$ and $\left\{b_{n}, n \geq 1\right\}$ be two sequences of positive numbers with $c_{n}=b_{n} / a_{n}$ and $b_{n} \uparrow$ o. Let $f(x)=x^{r} h(x)$, where $h(x)>0$ is a slowly varying function as $x \rightarrow \infty, 1<$ $r<2$. Define $N(x)=\operatorname{Card}\left\{n: c_{n} \leq x\right\}, x>0$. If

(i) $N(n)=O(f(n))$ for each $n \geq 1$;

(ii) $E f(|X|)<\infty$.

Then there exist $d_{n} \in \mathbf{R}, n=1,2, \ldots$, such that

$$
b_{n}^{-1} \sum_{i=1}^{n} a_{i} X_{i}-d_{n} \longrightarrow 0 \quad \text { a.s. }
$$

Proof. It is easy to verify that conditions (i)-(iii) of Theorem 23 hold under the conditions of Corollary 25. So Corollary 25 is true by Theorem 23 .

Corollary 27. Suppose that the conditions of Corollary 26 are satisfied. If $E X_{n}=0$ for each $n \geq 1$, then $b_{n}^{-1} \sum_{i=1}^{n} a_{i} X_{i} \rightarrow$ 0 a.s.

Proof. According to Corollary 26, we need only to prove (60). By $E X_{n}=0$ for each $n \geq 1$ and Lemma 12, we have

$$
\begin{aligned}
& \sum_{i=1}^{\infty} \frac{a_{i}\left|E X_{i}^{\left(c_{i}\right)}\right|}{b_{i}} \\
& \quad \leq \sum_{i=1}^{\infty} c_{i}^{-1}\left[c_{i} E I\left(\left|X_{i}\right|>c_{i}\right)+\left|E X_{i} I\left(\left|X_{i}\right| \leq c_{i}\right)\right|\right] \\
& \quad \leq \sum_{i=1}^{\infty} c_{i}^{-1}\left[E\left|X_{i}\right| I\left(\left|X_{i}\right|>c_{i}\right)+\left|E X_{i} I\left(\left|X_{i}\right|>c_{i}\right)\right|\right]
\end{aligned}
$$




$$
\begin{aligned}
& \leq 2 \sum_{i=1}^{\infty} c_{i}^{-1} E\left|X_{i}\right| I\left(\left|X_{i}\right|>c_{i}\right) \\
& \leq C \sum_{i=1}^{\infty} c_{i}^{-1} E|X| I\left(|X|>c_{i}\right) \\
& =C \sum_{k=1}^{\infty} \sum_{(k-1)<c_{i} \leq k} c_{i}^{-1} E|X| I\left(|X|>c_{i}>k-1\right) \\
& \leq C \sum_{k=2}^{\infty}(k-1)^{-1}(N(k)-N(k-1)) \\
& \quad \times \sum_{j=k}^{\infty} E|X| I(j \leq|X|<j+1) \\
& \leq C \sum_{j=2}^{\infty} \sum_{k=2}^{j} N(k)\left((k-1)^{-1}-k^{-1}\right) \\
& \quad \times E|X| I(j \leq|X|<j+1) .
\end{aligned}
$$

Since $r>1$, we can take $\delta>0$ such that $r-\delta>1$. By Lemma 13 and differential mean value theorem, we can obtain

$$
\begin{aligned}
& \sum_{k=2}^{j} N(k)\left((k-1)^{-1}-k^{-1}\right) \\
& \leq C \sum_{k=2}^{j} k^{r} h(k)\left((k-1)^{-1}-k^{-1}\right) \\
& \quad=C \sum_{k=2}^{j} k^{r-\delta}\left(k^{\delta} h(k)\right)\left((k-1)^{-1}-k^{-1}\right) \\
& \leq C j^{\delta} h(j) \sum_{k=2}^{j} k^{r-\delta-2} \\
& \leq C j^{\delta} h(j) \int_{2}^{j} x^{r-\delta-2} d x \leq C j^{r-1} h(j) .
\end{aligned}
$$

It is easily seen that $x^{r-1} h(x)$ is a quasimonotonically increasing function by Lemma 13 . Hence, we have by (75) and (76) that

$$
\begin{aligned}
& \sum_{i=1}^{\infty} \frac{a_{i}\left|E X_{i}^{\left(c_{i}\right)}\right|}{b_{i}} \\
& \quad \leq C \sum_{j=2}^{\infty} j^{r-1} h(j) E|X| I(j \leq|X|<j+1) \\
& \quad \leq C \sum_{j=2}^{\infty} E\left(|X|^{r-1} h(|X|)|X| I(j \leq|X|<j+1)\right)
\end{aligned}
$$

$$
\begin{aligned}
& =C \sum_{j=2}^{\infty} E\left(|X|^{r} h(|X|) I(j \leq|X|<j+1)\right) \\
& \leq \operatorname{CEf}(|X|)<\infty
\end{aligned}
$$

By Kronecker's lemma, we can get (60) immediately. The proof is complete.

\section{Conflict of Interests}

The authors declare that there is no conflict of interests regarding the publication of this paper.

\section{Acknowledgments}

The authors are most grateful to the Editor Jaume Giné and anonymous referees for careful reading of the paper and valuable suggestions which helped in improving an earlier version of this paper. This work is supported by the National Natural Science Foundation of China (11171001, 11201001), the Natural Science Foundation of Anhui Province (1208085QA03, 1308085QA03), Doctoral Research Start-Up Funds Projects of Anhui University, Applied Teaching Model Curriculum of Anhui University (XJYYXKC04), the Students Innovative Training Project of Anhui University (201310357004, cxcy2012003), and the Students Science Research Training Program of Anhui University (KYXL2012007, kyxl2013003).

\section{References}

[1] Q. Y. Wu, Probability Limit Theory Mixing Sequences, Sciences Press of China, Beijing, China, 2006.

[2] Y. S. Chow and H. Teicher, Probability Theory: Independence, Interchangeability, Martingales, Springer, New York, NY, USA, 2nd edition, 1988.

[3] K. Joag-Dev and F. Proschan, "Negative association of random variables, with applications," The Annals of Statistics, vol. 11, no. 1, pp. 286-295, 1983.

[4] T. K. Chandra and S. Ghosal, "Extensions of the strong law of large numbers of Marcinkiewicz and Zygmund for dependent variables," Acta Mathematica Hungarica, vol. 71, no. 4, pp. 327336, 1996.

[5] T. K. Chandra and S. Ghosal, "The strong law of large numbers for weighted averages under dependence assumptions," Journal of Theoretical Probability, vol. 9, no. 3, pp. 797-809, 1996.

[6] M.-H. Ko, T.-S. Kim, and Z. Lin, “The Hájeck-Rènyi inequality for the AANA random variables and its applications," Taiwanese Journal of Mathematics, vol. 9, no. 1, pp. 111-122, 2005.

[7] Y. Wang, J. Yan, F. Cheng, and C. Su, “The strong law of large numbers and the law of the iterated logarithm for product sums of NA and AANA random variables," Southeast Asian Bulletin of Mathematics, vol. 27, no. 2, pp. 369-384, 2003.

[8] D. Yuan and J. An, "Rosenthal type inequalities for asymptotically almost negatively associated random variables and applications," Science in China A, vol. 52, no. 9, pp. 1887-1904, 2009.

[9] X. J. Wang, S. H. Hu, and W. Z. Yang, "Convergence properties for asymptotically almost negatively associated sequence," Discrete Dynamics in Nature and Society, vol. 2010, Article ID 218380, 15 pages, 2010. 
[10] X. J. Wang, S. H. Hu, X. Q. Li, and W. Z. Yang, "Maximal inequalities and strong law of large numbers for AANA sequences," Communications of the Korean Mathematic Society, vol. 26, no. 1, pp. 151-161, 2011.

[11] W. Z. Yang, X. J. Wang, N. X. Ling, and S. H. Hu, "On complete convergence of moving average process for AANA sequence," Discrete Dynamics in Nature and Society, vol. 2012, Article ID 863931, 24 pages, 2012.

[12] X. Hu, G. Fang, and D. Zhu, "Strong convergence properties for asymptotically almost negatively associated sequence," Discrete Dynamics in Nature and Society, vol. 2012, Article ID 562838, 8 pages, 2012.

[13] A. T. Shen and R. C. Wu, "Strong and weak convergence for asymptotically almost negatively associated random variables," Discrete Dynamics in Nature and Society, vol. 2013, Article ID 235012, 7 pages, 2013.

[14] A. T. Shen and R. C. Wu, "Strong convergence for sequences of asymptotically almost negatively associated random variables," Stochastics, Article ID 775289, 2013.

[15] X. J. Wang, S. H. Hu, and W. Z. Yang, "Complete convergence for arrays of rowwise asymptotically almost negatively associated random variables," Discrete Dynamics in Nature and Society, vol. 2011, Article ID 717126, 11 pages, 2011.

[16] X. J. Wang, S. H. Hu, W. Z. Yang, and X. H. Wang, "On complete convergence of weighted sums for arrays of rowwise asymptotically almost negatively associated random variables," Abstract and Applied Analysis, vol. 2012, Article ID 315138, 15 pages, 2012.

[17] A. T. Shen, R. C. Wu, Y. Chen, and Y. Zhou, "Complete convergence of the maximum partial sums for arrays of rowwise of AANA random variables," Discrete Dynamics in Nature and Society, vol. 2013, Article ID 741901, 7 pages, 2013.

[18] P. Matuła, "A note on the almost sure convergence of sums of negatively dependent random variables," Statistics \& Probability Letters, vol. 15, no. 3, pp. 209-213, 1992.

[19] Q. Y. Wu, "A strong limit theorem for weighted sums of sequences of negatively dependent random variables," Journal of Inequalities and Applications, vol. 2010, Article ID 383805, 2010.

[20] A. T. Shen, "Some strong limit theorems for arrays of rowwise negatively orthant-dependent random variables," Journal of Inequalities and Applications, vol. 2011, article 93, 2011.

[21] S. Gan, "Strong stability of weighted sums of NA random variables," International Journal of Mathematics and Mathematical Sciences, no. 6, pp. 975-985, 2005.

[22] W. Feller, An Introduction To Probability and Its Applications, vol. 2, John Wiley, New York, NY, USA, 2nd edition, 1971.

[23] A. Adler, "On the nonexistence of a law of the iterated logarithm for weighted sums of identically distributed random variables," Journal of Applied Mathematics and Stochastic Analysis, vol. 3, no. 2, pp. 135-140, 1990.

[24] X. J. Wang, X. Q. Li, S. H. Hu, and W. Z. Yang, "Strong limit theorems for weighted sums of negatively associated random variables," Stochastic Analysis and Applications, vol. 29, no. 1, pp. $1-14,2011$. 


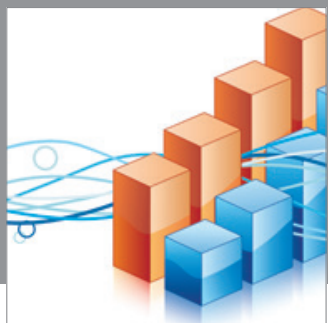

Advances in

Operations Research

mansans

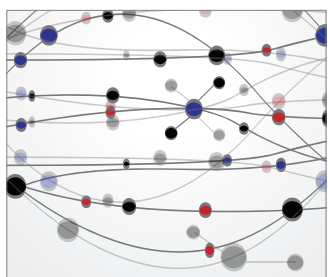

The Scientific World Journal
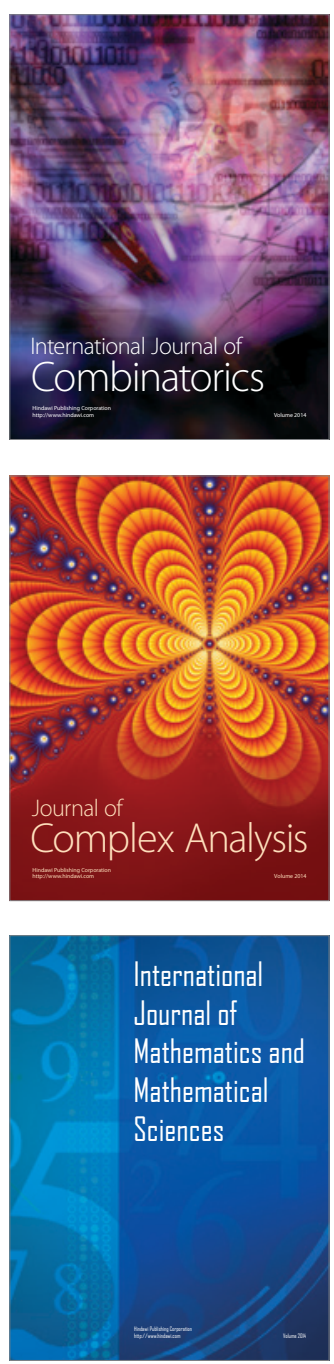
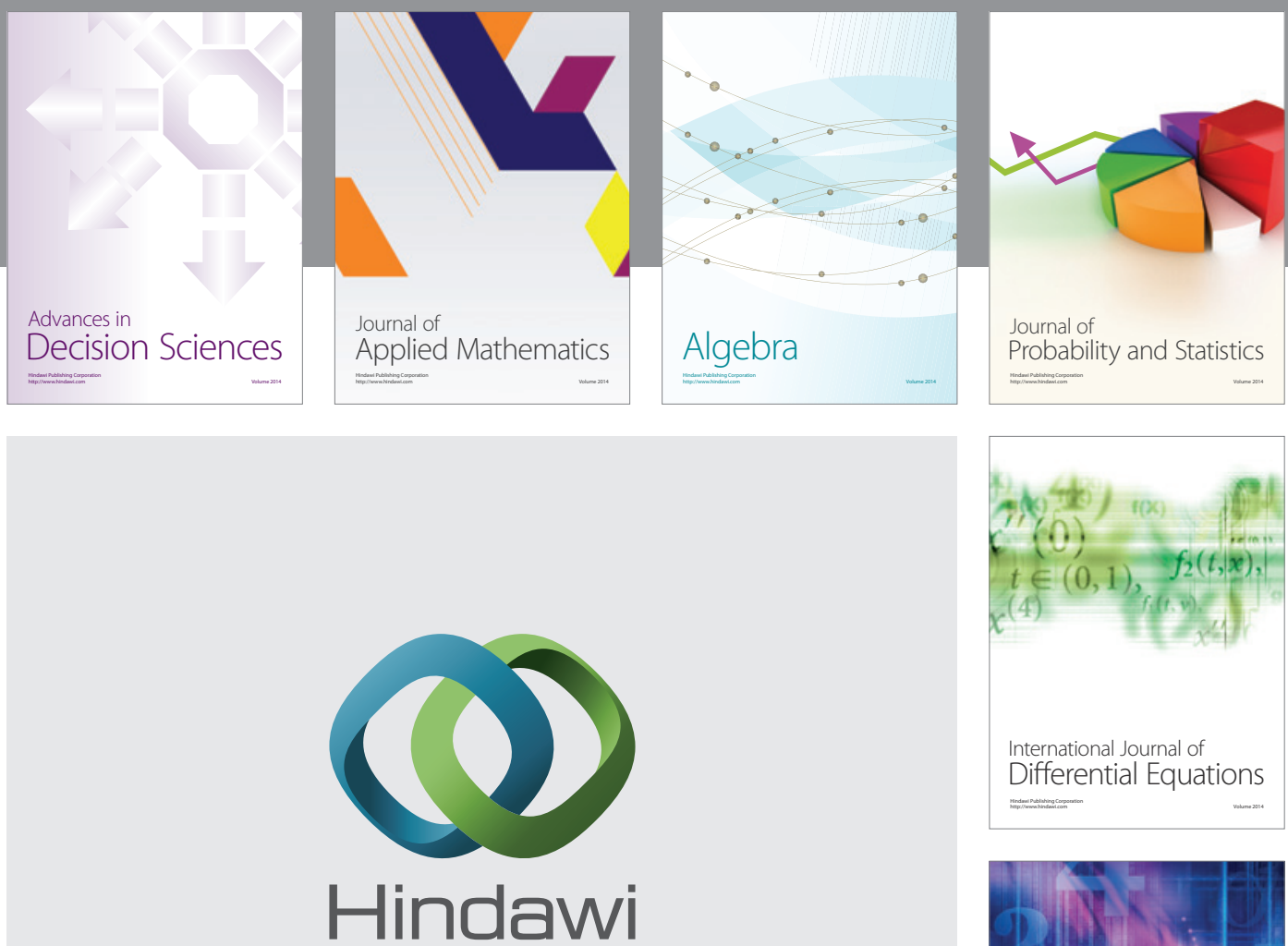

Submit your manuscripts at http://www.hindawi.com
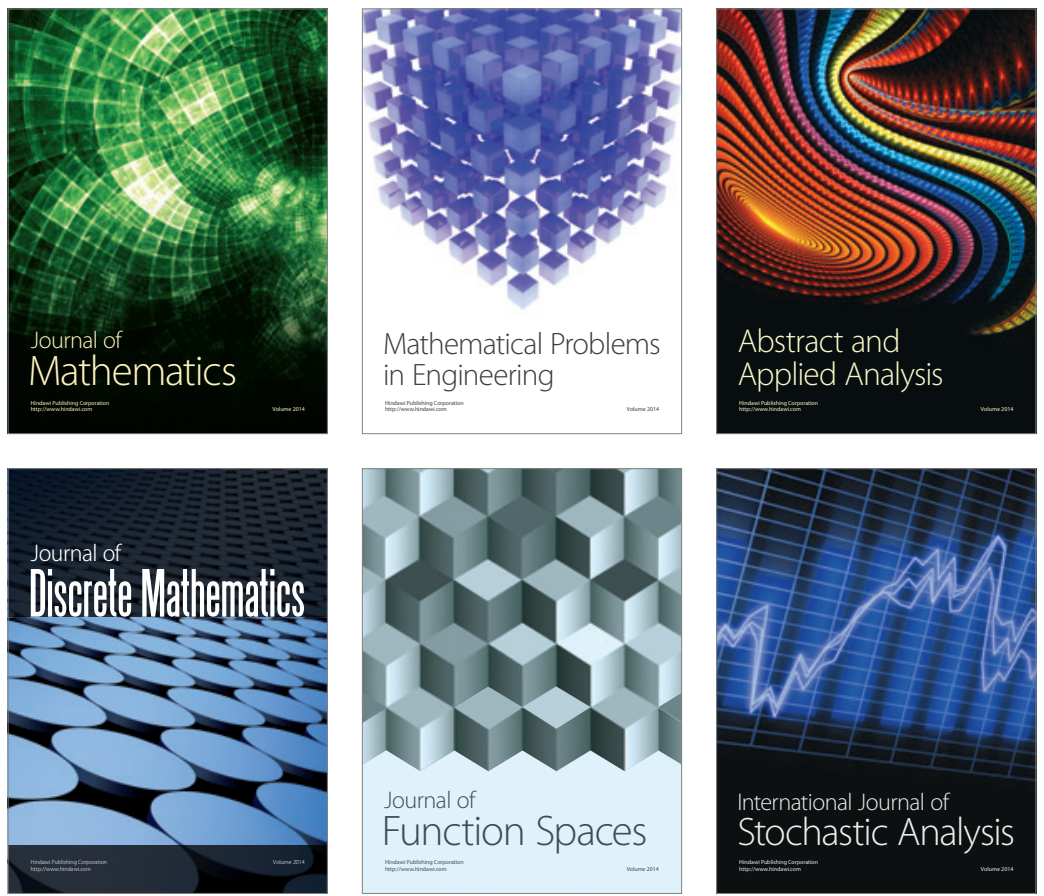

Journal of

Function Spaces

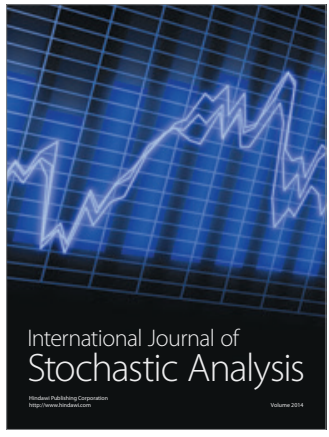

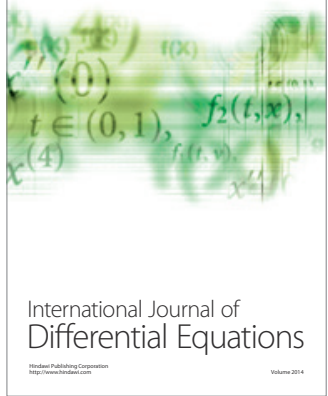
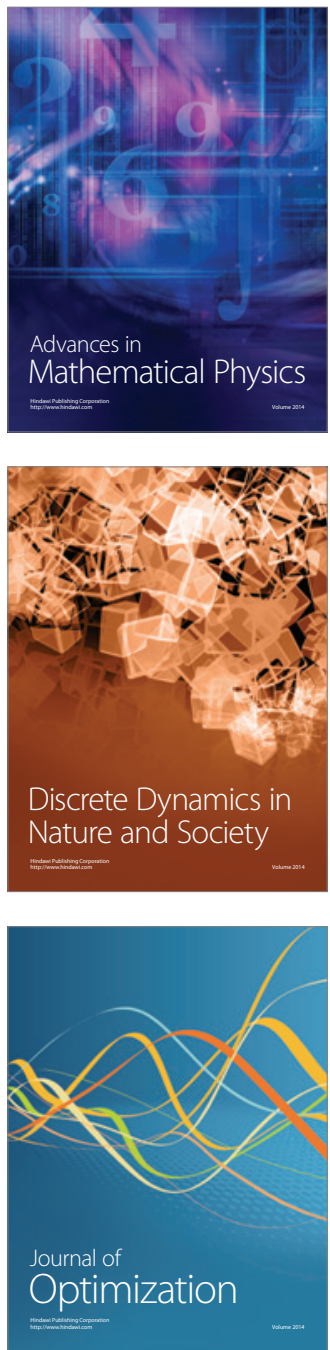